\title{
Para-Ricci-Like Solitons on Riemannian Manifolds with Almost Paracontact Structure and Almost Paracomplex Structure
}

\author{
Hristo Manev ${ }^{1, *,+(\mathbb{D})}$ and Mancho Manev ${ }^{1,2,+}+\mathbb{D}$ \\ 1 Department of Medical Informatics, Biostatistics and E-Learning, Faculty of Public Health, Medical \\ University of Plovdiv, 15A Vasil Aprilov Blvd, 4002 Plovdiv, Bulgaria; mmanev@uni-plovdiv.bg \\ 2 Department of Algebra and Geometry, Faculty of Mathematics and Informatics, University of Plovdiv Paisii \\ Hilendarski, 24 Tzar Asen St, 4000 Plovdiv, Bulgaria \\ * Correspondence: hristo.manev@mu-plovdiv.bg; Tel.: +359-887-440-560 \\ + These authors contributed equally to this work.
}

Citation: Manev, H.; Manev, M.

Para-Ricci-Like Solitons on

Riemannian Manifolds with Almost

Paracontact Structure and Almost Paracomplex Structure. Mathematics 2021, 9, 1704. https://doi.org/ $10.3390 /$ math 9141704

Academic Editor: George

Kaimakamis

Received: 25 June 2021

Accepted: 16 July 2021

Published: 20 July 2021

Publisher's Note: MDPI stays neutral with regard to jurisdictional claims in published maps and institutional affiliations.

Copyright: (C) 2021 by the authors. Licensee MDPI, Basel, Switzerland. This article is an open access article distributed under the terms and conditions of the Creative Commons Attribution (CC BY) license (https:/ / creativecommons.org/licenses/by/ $4.0 /)$.
Abstract: We introduce and study a new type of soliton with a potential Reeb vector field on Riemannian manifolds with an almost paracontact structure corresponding to an almost paracomplex structure. The special cases of para-Einstein-like, para-Sasaki-like and having a torse-forming Reeb vector field were considered. It was proved a necessary and sufficient condition for the manifold to admit a para-Ricci-like soliton, which is the structure that is para-Einstein-like. Explicit examples are provided in support of the proven statements.

Keywords: para-Ricci-like soliton; $\eta$-Ricci soliton; para-Einstein-like manifold; $\eta$-Einstein manifold; almost paracontact structure; almost paracomplex structure; Riemannian manifold; torse-forming vector field

MSC: 53C25; 53D15; 53C50; 53C44; 53D35; 70G45

\section{Introduction}

The concept of Ricci solitons was introduced in 1982 by R. S. Hamilton [1] as a special self-similar solution of the Ricci flow equation; it plays an important role in understanding its singularities. In other words, Ricci solitons represent a generalization of Einstein metrics on a Riemannian manifold, being generalized fixed points of the Ricci flow, considered a dynamical system. A detailed study on Riemannian Ricci solitons can be found in [2].

The study of Ricci solitons in contact Riemannian geometry began in 2008 by R. Sharma [3]. After that, the investigation of the geometric characteristics of Ricci solitons has continued on different types of almost contact metric manifolds (see [4-8]). The notion of the $\eta$-Ricci soliton, introduced by J. T. Cho and M. Kimura [9], is a generalization of the concept of the Ricci soliton. Investigations on $\eta$-Ricci solitons were made in [10-12], but in the context of paracontact geometry.

Recently, various geometers have extensively studied the pseudo-Riemannian properties of Ricci solitons as their generalizations. This interest is based on the growing interest of theoretical physicists in Ricci solitons and their relation with string theory. For further references on pseudo-Riemannian Ricci solitons, see [13-17].

The object of our focus is the geometry of Riemannian manifolds with an almost paracontact structure corresponding to an almost paracomplex structure. This type of structure here we call, briefly, a П-structure, and these manifolds Riemannian П-manifolds. In these manifolds, the induced almost product structure on the paracontact distribution is traceless, and the restriction on the paracontact distribution of the almost paracontact structure is an almost paracomplex structure. In [18], these manifolds are introduced and classified. Their investigation is continued in $[19,20]$.

In the present paper, due to the presence of two associated metrics, we introduce and study our generalization of the Ricci soliton compatible with the manifold structure, called 
the para-Ricci-like soliton. We characterize it in terms of some important types of manifolds under consideration - para-Einstein-like, para-Sasaki-like and having a torse-forming Reeb vector field. We provide two examples in support of the proven statements.

\section{The Main Results}

Theorem 1. Let $(\mathcal{M}, \phi, \xi, \eta, g)$ be a $(2 n+1)$-dimensional para-Sasaki-like Riemannian $\Pi$-manifold. Let $a, b, c, \lambda, \mu, v$ be constants satisfying the following conditions:

$$
a+\lambda=0, \quad b+\mu+1=0, \quad c+v-1=0 .
$$

Then, $(\mathcal{M}, \phi, \xi, \eta, g)$ admits a para-Ricci-like soliton with potential $\xi$ and constants $(\lambda, \mu, v)$, where $\lambda+\mu+v=2 n$, if and only if it is para-Einstein-like with constants $(a, b, c)$, where $a+b+c=-2 n$.

In particular, we obtain the following:

(i) $(\mathcal{M}, \phi, \xi, \eta, g)$ admits an $\eta$-Ricci soliton with potential $\xi$ and constants $(\lambda, 0,2 n-\lambda)$ if and only if $(\mathcal{M}, \phi, \xi, \eta, g)$ is para-Einstein-like with constants $(-\lambda,-1, \lambda-2 n+1)$.

(ii) $(\mathcal{M}, \phi, \xi, \eta, g)$ admits a shrinking Ricci soliton with potential $\xi$ and constants $(2 n, 0,0)$ if and only if $(\mathcal{M}, \phi, \xi, \eta, g)$ is para-Einstein-like with constants $(-2 n,-1,1)$.

(iii) $(\mathcal{M}, \phi, \xi, \eta, g)$ is $\eta$-Einstein with constants $(a, 0,-2 n-a)$ if and only if $(\mathcal{M}, \phi, \xi, \eta, g)$ admits a para-Ricci-like soliton with potential $\xi$ and constants $(-a,-1, a+2 n+1)$.

(iv) $(\mathcal{M}, \phi, \xi, \eta, g)$ is Einstein with constants $(2 n, 0,0)$ if and only if $(\mathcal{M}, \phi, \xi, \eta, g)$ admits a para-Ricci-like soliton with potential $\xi$ and constants $(2 n,-1,1)$.

Theorem 2. Let $(\mathcal{M}, \phi, \xi, \eta, g)$ be a Riemannian $\Pi$-manifold and $\xi$ be torse-forming with function $f$. Then, $(\mathcal{M}, \phi, \xi, \eta, g)$ admits a para-Ricci-like soliton with potential $\xi$ and constants $(\lambda, \mu, v)$ if and only if $(\mathcal{M}, \phi, \xi, \eta, g)$ is para-Einstein-like with constants $(a, b, c)$ satisfying the following conditions:

$$
a+\lambda+f=0, \quad b+\mu=0, \quad c+v-f=0,
$$

where $f$ is a constant.

In particular, we have the following:

(i) $(\mathcal{M}, \phi, \xi, \eta, g)$ admits an $\eta$-Ricci soliton with potential $\xi$ and constants $(\lambda, 0, v)$ if and only if $(\mathcal{M}, \phi, \xi, \eta, g)$ is $\eta$-Einstein with constants $(a, 0, c)$, where $a+c=-\lambda-v$.

(ii) $(\mathcal{M}, \phi, \xi, \eta, g)$ admits a Ricci soliton with potential $\xi$ and constants $(\lambda, 0,0)$ if and only if $(\mathcal{M}, \phi, \xi, \eta, g)$ is $\eta$-Einstein with constants $(-\lambda-f, 0, f)$.

(iii) $(\mathcal{M}, \phi, \xi, \eta, g)$ is Einstein with constants $(a, 0,0)$ if and only if $(\mathcal{M}, \phi, \xi, \eta, g)$ admits an $\eta$-Ricci soliton with potential $\xi$ and constants $(-a-f, 0, f)$.

\section{Riemannian П-Manifolds}

Let $(\mathcal{M}, \phi, \xi, \eta, g)$ be a Riemannian $\Pi$-manifold, namely, $\mathcal{M}$ is a differentiable odddimensional manifold, equipped with a Riemannian metric $g$ and an almost paracontact structure $(\phi, \xi, \eta)$, i.e., $\phi$ is a $(1,1)$-tensor field, $\xi$ is the characteristic vector field and $\eta$ is its dual 1-form, which satisfy the following:

$$
\begin{gathered}
\phi \xi=0, \quad \phi^{2}=I-\eta \otimes \xi, \quad \eta \circ \phi=0, \quad \eta(\xi)=1, \\
\operatorname{tr} \phi=0, \quad g(\phi x, \phi y)=g(x, y)-\eta(x) \eta(y),
\end{gathered}
$$

where $I$ is the identity transformation on $T \mathcal{N}([19,21])$. Consequently, we obtain the following equations:

$$
\begin{array}{ll}
g(\phi x, y)=g(x, \phi y), & g(x, \xi)=\eta(x), \\
g(\xi, \xi)=1, & \eta\left(\nabla_{x} \xi\right)=0,
\end{array}
$$

where $\nabla$ denotes the Levi-Civita connection of $g$. Here and further, $x, y, z$, w stand for arbitrary vector fields from $\mathfrak{X}(\mathcal{M})$ or vectors in $T \mathcal{M}$ at a fixed point of $\mathcal{M}$. 
The associated metric $\tilde{g}$ of $g$ on $(\mathcal{M}, \phi, \xi, \eta, g)$ is determined by the equality $\tilde{g}(x, y)=$ $g(x, \phi y)+\eta(x) \eta(y)$. Therefore, $\tilde{g}$ is an indefinite metric of signature $(n+1, n)$, which is compatible with $(\mathcal{M}, \phi, \xi, \eta, g)$ in the same way as $g$.

The Riemannian $\Pi$-manifolds are classified in [18]. This classification contains eleven basic classes, $\mathcal{F}_{1}, \mathcal{F}_{2}, \ldots, \mathcal{F}_{11}$, and it is made with respect to the $(0,3)$-tensor $F$ determined by the following:

$$
F(x, y, z)=g\left(\left(\nabla_{x} \phi\right) y, z\right) .
$$

The following basic properties of $F$ are valid:

$$
\begin{aligned}
& F(x, y, z)=F(x, z, y) \\
& =-F(x, \phi y, \phi z)+\eta(y) F(x, \xi, z)+\eta(z) F(x, y, \xi), \\
& \left(\nabla_{x} \eta\right) y=g\left(\nabla_{x} \xi, y\right)=-F(x, \phi y, \xi) .
\end{aligned}
$$

The intersection of the basic classes is denoted by $\mathcal{F}_{0}$, which is the special class determined by the condition $F=0$.

The one-forms associated with $F$ (also known as Lee forms) are defined by the following:

$$
\theta=g^{i j} F\left(e_{i}, e_{j}, \cdot\right), \quad \theta^{*}=g^{i j} F\left(e_{i}, \phi e_{j}, \cdot\right), \quad \omega=F(\xi, \xi, \cdot),
$$

where $\left(g^{i j}\right)$ is the inverse matrix of $\left(g_{i j}\right)$ of $g$ with respect to a basis $\left\{\xi ; e_{i}\right\}(i=1,2, \ldots, 2 n)$ of $T_{p} \mathcal{M}, p \in \mathcal{M}$.

\subsection{Para-Sasaki-Like Manifolds}

In [22], the class of para-Sasaki-like spaces is defined in the set of Riemannian Пmanifolds obtained from a specific cone construction. The considered para-Sasaki-like spaces are determined by the following:

$$
\begin{aligned}
\left(\nabla_{x} \phi\right) y & =-g(x, y) \xi-\eta(y) x+2 \eta(x) \eta(y) \xi \\
& =-g(\phi x, \phi y) \xi-\eta(y) \phi^{2} x
\end{aligned}
$$

Remark 1. The Riemannian П-manifolds of para-Sasaki-like type form a subclass of the basic class $\mathcal{F}_{4}$ from [18] and their Lee forms are $\theta=-2 n \eta$ and $\theta^{*}=\omega=0$.

In [22], the truthfulness of the following identities is proved by the following:

$$
\begin{array}{ll}
\nabla_{x} \xi=\phi x, & \left(\nabla_{x} \eta\right)(y)=g(x, \phi y), \\
R(x, y) \xi=-\eta(y) x+\eta(x) y, & R(\xi, y) \xi=\phi^{2} y, \\
\rho(x, \xi)=-2 n \eta(x), & \rho(\xi, \xi)=-2 n,
\end{array}
$$

where $R$ and $\rho$ denote the curvature tensor and the Ricci tensor, respectively.

\subsection{Para-Einstein-Like Manifolds}

Definition 1. A Riemannian П-manifold $(\mathcal{M}, \phi, \xi, \eta, g)$ is said to be para-Einstein-like with constants $(a, b, c)$ if its Ricci tensor $\rho$ satisfies the following:

$$
\rho=a g+b \tilde{g}+c \eta \otimes \eta
$$

In particular, the manifold is known to be called an $\eta$-Einstein manifold and an Einstein manifold when $b=0$ and $b=c=0$, respectively.

Using (10), we obtain the corresponding scalar curvature as follows:

$$
\tau=(2 n+1) a+b+c
$$

Remark 2. The Reeb vector field $\xi$ of a para-Einstein-like manifold $(\mathcal{M}, \phi, \xi, \eta, g)$ is an eigenvector of the Ricci operator $Q=\rho^{\sharp}$, i.e., $g(Q x, y)=\rho(x, y)$, corresponding to the eigenvalue $a+b+c$. 
Proposition 1. The Ricci tensor $\rho$ of a para-Einstein-like manifold $(\mathcal{M}, \phi, \xi, \eta, g)$ has the following properties:

$$
\begin{gathered}
\rho(\phi x, \phi y)=\rho(x, y)-(a+b+c) \eta(x) \eta(y), \\
\rho(\phi x, y)=\rho(x, \phi y), \quad \rho(\phi x, \xi)=0, \\
\rho(x, \xi)=(a+b+c) \eta(x), \quad \rho(\xi, \xi)=a+b+c, \\
\left(\nabla_{x} \rho\right)(y, z)=b g\left(\left(\nabla_{x} \phi\right) y, z\right) \\
\quad+(b+c)\left\{g\left(\nabla_{x} \xi, y\right) \eta(z)+g\left(\nabla_{x} \xi, z\right) \eta(y)\right\} .
\end{gathered}
$$

Proof. We confirm the validity of the proposition by applying (3) and (4) in (10).

Proposition 2. Let $(\mathcal{M}, \phi, \xi, \eta, g)$ be a $(2 n+1)$-dimensional Riemannian $\Pi$-manifold, which is para-Sasaki-like and para-Einstein-like with constants $(a, b, c)$. Then, we have the following:

$$
\begin{gathered}
a+b+c=-2 n, \quad \tau=2 n(a-1), \\
\left(\nabla_{x} \rho\right)(y, z)=(b+c)\{g(x, \phi y) \eta(z)+g(x, \phi z) \eta(y)\}, \\
-b\{g(\phi x, \phi y) \eta(z)+g(\phi x, \phi z) \eta(y)\}, \\
a=-2 n-\frac{1}{2 n}\left(\operatorname{div}^{*} \rho\right)(\xi), \quad b=-\frac{1}{2 n}(\operatorname{div} \rho)(\xi), \\
c=\frac{1}{2 n}\left\{(\operatorname{div} \rho)(\xi)+\left(\operatorname{div}^{*} \rho\right)(\xi)\right\},
\end{gathered}
$$

where div and $\operatorname{div}^{*}$ stand for the divergence with respect to $g$ and $\tilde{g}$, respectively.

Proof. Using (11) and the last equalities of (9) and (12), we obtain (14). The next expression (15) follows from (8) and (13). We obtain the assertions in (16) by taking the traces over $x$ and $y$ in (15) with respect to both metrics, using $\tilde{g}^{i j}=\phi_{k}^{j} g^{i k}+\xi^{i} \xi^{j}$.

Corollary 1. Let $(\mathcal{M}, \phi, \xi, \eta, g)$ be a para-Sasaki-like para-Einstein-like manifold with constants $(a, b, c)$. Then:

(i) A necessary and sufficient condition $(\mathcal{M}, \phi, \xi, \eta, g)$ to be scalar-flat is $a=1$.

(ii) A necessary and sufficient condition $(\mathcal{M}, \phi, \xi, \eta, g)$ to be Ricci-symmetric is to be an Einstein manifold.

(iii) The Ricci tensor of $(\mathcal{M}, \phi, \xi, \eta, g)$ is $\eta$-parallel and parallel along $\xi$.

(iv) A necessary and sufficient condition $(\mathcal{M}, \phi, \xi, \eta, g)$ to be $\eta$-Einstein is div $Q \in \operatorname{ker} \eta$.

(v) A necessary and sufficient condition $(\mathcal{M}, \phi, \xi, \eta, g)$ to be Einstein is $\operatorname{div} Q, \operatorname{div}^{*} Q \in \operatorname{ker} \eta$.

Proof. It is easy to check the validity of (i) and (ii), bearing in mind (14) and (15), respectively. The truthfulness of (iii) comes from $\left.(\nabla \rho)\right|_{\text {ker } \eta}=0$ and $\nabla_{\xi} \rho=0$, which are corollaries of (15). Similarly, we obtain that (iv) and (v) are valid, using the value of $b$ in (16) and the identities $\left(\nabla_{x} \rho\right)(y, z)=g\left(\left(\nabla_{x} Q\right) y, z\right)$ and $(\operatorname{div} \rho)(z)=g(\operatorname{div} Q, z)$.

Moreover, using (14), we obtain that each Einstein para-Sasaki-like $(\mathcal{M}, \phi, \xi, \eta, g)$ has a scalar curvature $\tau=-2 n(2 n+1)$.

\subsection{Riemannian П-Manifolds with a Torse-Forming Reeb Vector Field}

It is known that a vector field $\xi$ is called torse-forming if $\nabla_{x} \xi=f x+\alpha(x) \xi$ is valid for a smooth function $f$ and a one-form $\alpha$ on the manifold. The one-form $\alpha$ on a Riemannian $\Pi$-manifold $(\mathcal{M}, \phi, \xi, \eta, g)$ is determined by $\alpha=-f \eta$, taking into account the last equality in (4). Then, we have the following equivalent equalities:

$$
\nabla_{x} \xi=f \phi^{2} x, \quad\left(\nabla_{x} \eta\right)(y)=f g(\phi x, \phi y) .
$$

In the further investigations, we omit the trivial case when $f=0$. 
Proposition 3. Each $(\mathcal{M}, \phi, \xi, \eta, g)$ with torse-forming $\xi$ belongs to the class $\mathcal{F}_{1} \oplus \mathcal{F}_{2} \oplus \mathcal{F}_{3} \oplus$ $\mathcal{F}_{5} \oplus \mathcal{F}_{6} \oplus \mathcal{F}_{10}$. Moreover, among the basic classes, only $\mathcal{F}_{5}$ can contain such a manifold.

Proof. Taking into account (6) and (7), equalities (17) imply $\theta^{*}(\xi)=-2 n f$ and $\theta(\xi)=$ $\omega=0$. Therefore, bearing in mind the components of $F$ in the basic classes $\mathcal{F}_{i}$, given in [19], we obtain the statement.

As a result of the latter proposition, a manifold $(\mathcal{M}, \phi, \xi, \eta, g) \in \mathcal{F}_{5}$ with torse-forming $\xi$ is determined by the following:

$$
\left(\nabla_{x} \phi\right) y=-f\{g(x, \phi y) \xi+\eta(y) \phi x\} .
$$

Proposition 4. The Reeb vector field $\xi$ of each para-Sasaki-like manifold $(\mathcal{M}, \phi, \xi, \eta, g)$ is not torse-forming.

Proof. Taking into account Remark 1 and Proposition 3, we prove the assertion.

Corollary 2. Let a Riemannian $\Pi$-manifold $(\mathcal{M}, \phi, \xi, \eta, g)$ with torse-forming $\xi$ be para-Einsteinlike. Then, a necessary and sufficient condition $(\mathcal{M}, \phi, \xi, \eta, g)$ to be Ricci-symmetric is to be an Einstein manifold.

Proof. Similar to (ii) of Corollary 1, we obtain the statement, using (13) and (17).

\section{Proofs of Theorem 1 and Theorem 2}

A Ricci soliton is a pseudo-Riemannian manifold $(M, g)$, which admits a smooth non-zero vector field $v$ on $M$ such that the following holds [1]:

$$
\rho=-\frac{1}{2} \mathcal{L}_{v} g-\lambda g
$$

where $\mathcal{L}$ stands for the Lie derivative and $\lambda$ is a constant. In particular, a Ricci soliton with negative, zero or positive $\lambda$ is called shrinking, steady or expanding, respectively [23].

The authors of [9] made a generalization of the Ricci soliton on an almost contact metric manifold $(M, \varphi, \xi, \eta, g)$ called an $\eta$-Ricci soliton determined by $\rho=-\frac{1}{2} \mathcal{L}_{v} g-\lambda g-v \eta \otimes \eta$, where $v$ is also a constant.

Due to the presence of two associated metrics on almost contact B-metric manifolds, a subsequent generalization of the Ricci soliton and the $\eta$-Ricci soliton is presented in [17], called a Ricci-like soliton. Here, we introduce our generalization in terms of Riemannian $\Pi$-manifolds as follows.

Definition 2. A Riemannian $\Pi$-manifold $(\mathcal{M}, \phi, \xi, \eta, g)$ is called a para-Ricci-like soliton with potential vector field $\xi$ and constants $(\lambda, \mu, v)$ if its Ricci tensor $\rho$ satisfies the following:

$$
\rho=-\frac{1}{2} \mathcal{L}_{\xi} g-\lambda g-\mu \tilde{g}-v \eta \otimes \eta .
$$

Taking into account (19) and

$$
\left(\mathcal{L}_{\xi} g\right)(x, y)=g\left(\nabla_{x} \xi, y\right)+g\left(x, \nabla_{y} \xi\right)
$$

we obtain the scalar curvature

$$
\tau=-\operatorname{div} \xi-(2 n+1) \lambda-\mu-v .
$$

Proposition 5. Let $(\mathcal{M}, \phi, \xi, \eta, g)$ be a para-Einstein-like manifold with constants $(a, b, c)$, and let $(\mathcal{M}, \phi, \xi, \eta, g)$ admit a para-Ricci-like soliton with potential $\xi$ and constants $(\lambda, \mu, v)$. Then, we have the following:

(i) $a+b+c=-\lambda-\mu-v$; 
(ii) $\nabla_{\xi} \xi=0$, i.e., $\xi$ is geodesic;

(iii) $\left(\nabla_{\xi} \phi\right) \xi=0, \nabla_{\xi} \eta=0, \omega=0$;

(iv) $\left(\nabla_{\xi} \rho\right)(y, z)=b g\left(\left(\nabla_{\xi} \phi\right) y, z\right)$.

Proof. By virtue of (10), (19) and (20), we obtain the following:

$$
\begin{aligned}
g\left(\nabla_{x} \xi, y\right)+g\left(\nabla_{y} \xi, x\right)=-2\{ & (a+\lambda) g(x, y)+(b+\mu) g(x, \phi y) \\
+ & (b+c+\mu+v) \eta(x) \eta(y)\} .
\end{aligned}
$$

By substituting $y$ for $\xi$ in the latter equality, we prove the truthfulness of (i) and (ii). The validity of (iii) comes from (5)-(7). Finally, (13) and $\nabla_{\xi} \xi=0$ yield (iv).

Corollary 3. Let $(\mathcal{M}, \phi, \xi, \eta, g)$ be a para-Einstein-like manifold with constants $(a, b, c)$, and let $(\mathcal{M}, \phi, \xi, \eta, g)$ admit a para-Ricci-like soliton with potential $\xi$ and constants $(\lambda, \mu, v)$. Then, we have the following:

(i) $(\mathcal{M}, \phi, \xi, \eta, g)$ does not belong to $\mathcal{F}_{11}$ or to a direct sum of $\mathcal{F}_{11}$ with other basic classes.

(ii) A necessary and sufficient condition for the Ricci tensor of $(\mathcal{M}, \phi, \xi, \eta, g)$ to be parallel along $\xi$ is for $(\mathcal{M}, \phi, \xi, \eta, g)$ not to belong to $\mathcal{F}_{10}$ or to a direct sum of $\mathcal{F}_{10}$ with other basic classes.

(iii) A necessary and sufficient condition for the Ricci tensor of $(\mathcal{M}, \phi, \xi, \eta, g)$ to be parallel along $\xi$ is for $(\mathcal{M}, \phi, \xi, \eta, g)$ to be $\eta$-Einstein.

Proof. The characterization of the basic classes $\mathcal{F}_{i}$ by the components of $F$, given in [19], and the assertions (iii) and (iv) in Proposition 5, complete the proof of the corollary.

Using the expression of $\nabla_{x} \xi$ from (9) in (20) and (19), we obtain that (19) takes the following form:

$$
\rho=-\lambda g-(1+\mu) \tilde{g}+(1-v) \eta \otimes \eta,
$$

which coincides with (10) under conditions (1) in Theorem A. The equality $a+b+c=-2 n$ comes from (14), whereas $\lambda+\mu+v=2 n$ is a result of (21) for $\rho(x, \xi)$ and the corresponding formula from (9). Therefore, the main assertion in Theorem 1 is proved.

We obtain the truthfulness of the assertions (i), (ii), (iii) and (iv) in Theorem A as corollaries of the main statement for the cases $\mu=0, \mu=v=0, b=0$ and $b=c=0$, respectively. This completes the proof of Theorem 1 .

Now, we focus our considerations on Theorem 2. Let $\xi$ on a Riemannian $\Pi$-manifold $(\mathcal{M}, \phi, \xi, \eta, g)$ be torse-forming with function $f$, and let $(\mathcal{M}, \phi, \xi, \eta, g)$ admit a para-Ricci-like soliton with potential $\xi$ and constants $(\lambda, \mu, v)$. Taking into account (17), (19) and (20), we obtain the form of the Ricci tensor of $(\mathcal{M}, \phi, \xi, \eta, g)$ as follows:

$$
\rho=-(\lambda+f) g-\mu \tilde{g}-(v-f) \eta \otimes \eta .
$$

Bearing in mind (10), the latter identity shows that a necessary and sufficient condition for $(\mathcal{M}, \phi, \xi, \eta, g)$ to be para-Einstein-like is for $f$ to be a constant. Then, the main statements (2) in Theorem B are valid.

Vice versa, let $(\mathcal{M}, \phi, \xi, \eta, g)$ have a torse-forming vector field $\xi$ with function $f$ and let $(\mathcal{M}, \phi, \xi, \eta, g)$ be para-Einstein-like with constants $(a, b, c)$. Applying (17) and (10) in (20) and (19) for $(\lambda, \mu, v)$, we have that (19) is satisfied if and only if (2) holds. This is true when $f$ is a constant.

We obtain the truthfulness of the assertions (i), (ii) and (iii) in Theorem B as corollaries of the main statement for the cases $b=\mu=0, b=\mu=v=0$ and $b=c=\mu=0$, respectively. This completes the proof of Theorem 2 . 


\section{Some Consequences of the Main Theorems}

Proposition 6. Let $(\mathcal{M}, \phi, \xi, \eta, g)$ be a para-Einstein-like manifold with constants $(a, b, c)$, and let $(\mathcal{M}, \phi, \xi, \eta, g)$ admit a para-Ricci-like soliton with potential $\xi$ and constants $(\lambda, \mu, v)$. Then $f$ is determined by the following:

$$
f=\varepsilon \sqrt{-\frac{a+b+c}{2 n}}=\varepsilon \sqrt{\frac{\lambda+\mu+v}{2 n}}, \quad \varepsilon= \pm 1 .
$$

Proof. Using the form of $\nabla \xi$ in (17), we obtain the following:

$$
R(x, y) \xi=f^{2}\{\eta(x) y-\eta(y) x\}, \quad \rho(x, \xi)=-2 n f^{2} \eta(x) .
$$

We have that $-2 n f^{2}=a+b+c$ from (23) and (12). This result and (i) of Proposition 5 imply (22).

Corollary 4. The $\xi$-sections $\alpha=\operatorname{span}\{x, \xi\}$ of a Riemannian $\Pi$-manifold $(\mathcal{M}, \phi, \xi, \eta, g)$ with a torse-forming potential $\xi$ and constant $f$ have constant negative sectional curvatures $k(\alpha)=-f^{2}$.

Proof. The form of the curvature tensor $R$ in (23) completes the proof of the corollary.

Corollary 5. A necessary and sufficient condition for $(\mathcal{M}, \phi, \xi, \eta, g)$ to be an Einstein manifold with a negative scalar curvature $\tau$ is for $(\mathcal{M}, \phi, \xi, \eta, g)$ to admit an $\eta$-Ricci soliton with potential $\xi$ and constants $(\lambda=-\tau /(2 n+1)-f, \mu=0, v=f)$, where $f$ is the constant for the torse-forming vector field $\xi$ and $f=\varepsilon \sqrt{-\tau /(2 n(2 n+1))}, \varepsilon= \pm 1$.

Proof. The expression of $\tau$ in (11) for an Einstein manifold, Proposition 6 and assertion (iii) of Theorem 2 complete the proof of this corollary.

By virtue of Proposition 3, we conclude the following.

Proposition 7. Let $(\mathcal{M}, \phi, \xi, \eta, g) \in \mathcal{F}_{5} \backslash \mathcal{F}_{0}$ be a para-Einstein-like manifold with constants $(a, b, c)$, and let $(\mathcal{M}, \phi, \xi, \eta, g)$ admit a para-Ricci-like soliton with potential $\xi$ and constants $(\lambda, \mu, v)$. Then, we have the following:

(i) A necessary and sufficient condition for $(\mathcal{M}, \phi, \xi, \eta, g)$ to be Ricci-symmetric is for ( $\mathcal{M}, \phi, \xi$, $\eta, g)$ to be an Einstein manifold.

(ii) The Ricci tensor of $(\mathcal{M}, \phi, \xi, \eta, g)$ is $\eta$-parallel and parallel along $\xi$.

Proof. Taking into account (13), (17), (18) and (2), we obtain the following:

$$
\begin{gathered}
\left(\nabla_{x} \rho\right)(y, z)=(a+\lambda)\{-(b+c)\{g(\phi x, \phi y) \eta(z)+g(\phi x, \phi z) \eta(y)\} \\
+b\{g(x, \phi y) \eta(z)+g(x, \phi z) \eta(y)\}\} .
\end{gathered}
$$

Using the latter result and following the proof for (ii) and (iii) in Corollary 1, we establish the truthfulness of the statements.

\section{Examples}

\subsection{Example 1}

In [22], a 5-dimensional Lie group $G$ is considered with a basis of left-invariant vector fields $\left\{e_{0}, \ldots, e_{4}\right\}$ and the corresponding Lie algebra is determined for $p, q \in \mathbb{R}$ as follows:

$$
\begin{array}{ll}
{\left[e_{0}, e_{1}\right]=p e_{2}-e_{3}+q e_{4},} & {\left[e_{0}, e_{2}\right]=-p e_{1}-q e_{3}-e_{4},} \\
{\left[e_{0}, e_{3}\right]=-e_{1}+q e_{2}+p e_{4},} & {\left[e_{0}, e_{4}\right]=-q e_{1}-e_{2}-p e_{3} .}
\end{array}
$$


In the same paper, $G$ is equipped with an invariant Riemannian $\Pi$-structure $(\phi, \xi, \eta, g)$ as follows:

$$
\begin{aligned}
& g\left(e_{0}, e_{0}\right)=g\left(e_{1}, e_{1}\right)=g\left(e_{2}, e_{2}\right)=g\left(e_{3}, e_{3}\right)=g\left(e_{4}, e_{4}\right)=1, \\
& g\left(e_{i}, e_{j}\right)=0, \quad i, j \in\{0,1, \ldots, 4\}, i \neq j, \\
& \xi=e_{0}, \quad \phi e_{1}=e_{3}, \quad \phi e_{2}=e_{4}, \quad \phi e_{3}=e_{1}, \quad \phi e_{4}=e_{2} .
\end{aligned}
$$

It is proved that the constructed manifold $(G, \phi, \xi, \eta, g)$ is a para-Sasaki-like manifold. Taking into account (24) and (25), we compute the components $R_{i j k l}=R\left(e_{i}, e_{j}, e_{k}, e_{l}\right)$ of the curvature tensor and the components $\rho_{i j}=\rho\left(e_{i}, e_{j}\right)$ of the Ricci tensor. The non-zero of them are determined by the following equalities and their well-known symmetries and antisymmetries:

$$
\begin{aligned}
& R_{0110}=R_{0220}=R_{0330}=R_{0440}=-1, \\
& R_{1234}=R_{1432}=R_{1331}=R_{2442}=1, \quad \rho_{00}=-4 .
\end{aligned}
$$

Therefore, using (10), we establish that the constructed manifold is $\eta$-Einstein with the following constants:

$$
(a, b, c)=(0,0,-4)
$$

Now, we check whether $(G, \phi, \xi, \eta, g)$ admits a Ricci-like soliton with potential $\xi$. By virtue of $(25)$ and $(20)$, we obtain the components $\left(\mathcal{L}_{\xi} g\right)_{i j}=\left(\mathcal{L}_{\xi \xi} g\right)\left(e_{i}, e_{j}\right)$, and the non-zero of them are the following:

$$
\left(\mathcal{L}_{\xi} g\right)_{13}=\left(\mathcal{L}_{\xi} g\right)_{24}=\left(\mathcal{L}_{\xi} g\right)_{31}=\left(\mathcal{L}_{\xi} g\right)_{42}=2 .
$$

Afterwards, using (25), (26) and (28), we obtain that (19) is satisfied for the following:

$$
(\lambda, \mu, v)=(0,-1,5)
$$

Therefore, the manifold $(G, \phi, \xi, \eta, g)$ admits a para-Ricci-like soliton with potential $\xi$.

Remark 3. The constructed five-dimensional para-Sasaki-like manifold $(G, \phi, \xi, \eta, g)$ is $\eta$-Einstein with constants given in (27) and $(G, \phi, \xi, \eta, g)$ admits a para-Ricci-like soliton with potential $\xi$ and constants determined in (29). Moreover, these constants satisfy (1); therefore, this example supports the assertion (iii) of Theorem 1.

\subsection{Example 2}

In [20], an example of a three-dimensional Riemannian $\Pi$-manifold belonging to the basic class $\mathcal{F}_{5}$ is considered. The constructed manifold is denoted by $(L, \phi, \xi, \eta, g)$, where $L$ is a Lie group with a basis $\left\{e_{0}, e_{1}, e_{2}\right\}$ of left invariant vector fields. The corresponding Lie algebra and the Riemannian $\Pi$-structure are defined by the following:

$$
\begin{gathered}
{\left[e_{0}, e_{1}\right]=p e_{1}, \quad\left[e_{0}, e_{2}\right]=p e_{2}, \quad\left[e_{1}, e_{2}\right]=0, \quad p \in \mathbb{R},} \\
\phi e_{0}=0, \quad \phi e_{1}=e_{2}, \quad \phi e_{2}=e_{1}, \quad \xi=e_{0}, \\
\eta\left(e_{0}\right)=1, \quad \eta\left(e_{1}\right)=\eta\left(e_{2}\right)=0, \\
g\left(e_{0}, e_{0}\right)=g\left(e_{1}, e_{1}\right)=g\left(e_{2}, e_{2}\right)=1, \\
g\left(e_{0}, e_{1}\right)=g\left(e_{0}, e_{2}\right)=g\left(e_{1}, e_{2}\right)=0 .
\end{gathered}
$$

In the same paper, it is obtained that $(L, \phi, \xi, \eta, g)$ is Einstein with the following constants:

$$
(a, b, c)=\left(-2 p^{2}, 0,0\right) \text {. }
$$

Moreover, it is easy to check that $\xi$ is a torse-forming vector field with the following constant:

$$
f=-p
$$


By virtue of (30) and (20), we obtain that the components $\left(\mathcal{L}_{\xi g}\right)_{i j}$ and the non-zero of them are the following:

$$
\left(\mathcal{L}_{\xi} g\right)_{11}=\left(\mathcal{L}_{\xi} g\right)_{22}=-2 p .
$$

After that, using (30) and (33), we have that (19) is satisfied for the following:

$$
(\lambda, \mu, v)=\left(p+2 p^{2}, 0,-p\right) .
$$

Therefore, the manifold $(L, \phi, \xi, \eta, g)$ admits a para-Ricci-like soliton with torseforming potential $\xi$.

Remark 4. The constructed three-dimensional Riemannian $\Pi$-manifold $(L, \phi, \xi, \eta, g)$ is Einstein with constants given in (31), and $(L, \phi, \xi, \eta, g)$ admits an $\eta$-Ricci soliton with potential $\xi$ and constants determined in (32) and (34). Moreover, these constants satisfy (2); therefore, this example supports the assertion (iii) of Theorem 2.

Author Contributions: Conceptualization, H.M. and M.M.; methodology, H.M. and M.M.; software, H.M. and M.M.; validation, H.M. and M.M.; formal analysis, H.M. and M.M.; investigation, H.M. and M.M.; resources, H.M. and M.M.; data curation, H.M. and M.M.; writing—original draft preparation, H.M. and M.M.; writing - review and editing, H.M. and M.M.; visualization, H.M. and M.M.; supervision, H.M. and M.M.; project administration, H.M. and M.M.; funding acquisition, H.M. and M.M. Both authors have read and agreed to the published version of the manuscript.

Funding: The research of H.M. is partially supported by the National Scientific Program "Young Researchers and Post-Doctorants" and the project MU21-FMI-008 of the Scientific Research Fund, University of Plovdiv Paisii Hilendarski. The research of M.M. is partially supported by projects MU21-FMI-008 and FP21-FMI-002 of the Scientific Research Fund, University of Plovdiv Paisii Hilendarski.

Institutional Review Board Statement: Not applicable.

Informed Consent Statement: Not applicable.

Data Availability Statement: Data are contained within the article.

Conflicts of Interest: The funders had no role in the design of the study; in the collection, analyses, or interpretation of data; in the writing of the manuscript, or in the decision to publish the results.

\section{References}

1. Hamilton, R.S. Three-manifolds with positive Ricci curvature. J. Differ. Geom. 1982, 17, 255-306. [CrossRef]

2. Cao, H.-D. Recent progress on Ricci solitons. Adv. Lect. Math. 2009, 11, 1-38.

3. Sharma, R. Certain results on K-contact and $(\kappa, \mu)$-contact manifolds. J. Geom. 2008, 89, 138-147. [CrossRef]

4. Ayar, G.; Yıldırım, M. $\eta$-Ricci solitons on nearly Kenmotsu manifolds. Asian-Eur. J. Math. 2019, 6, 2040002. [CrossRef]

5. Bagewadi, C.S.; Ingalahalli, G. Certain results on Ricci solitons in trans-Sasakian manifolds. J. Math. 2013, 2013, 787408. [CrossRef]

6. Gălin, C.; Crasmareanu, M. From the Eisenhart problem to Ricci solitons f-Kenmotsu manifolds. Bull. Malays. Math. Sci. Soc. 2010, 33, 361-368.

7. Ingalahalli, G.; Bagewadi, C.S. Ricci solitons in $\alpha$-Sasakian manifolds. Int. Sch. Res. Notices Geometry 2012, 2012, 421384. [CrossRef]

8. Nagaraja, H.G.; Premalatha, C.R. Ricci solitons in Kenmotsu manifolds. J. Math. Anal. 2012, 3, $18-24$.

9. Cho, J.T.; Kimura, M. Ricci solitons and real hypersurfaces in a complex space form. Tôhoku Math. J. 2009, 61, 205-212. [CrossRef]

10. Blaga, A.M. $\eta$-Ricci solitons on para-Kenmotsu manifolds. Balkan J. Geom. Appl. 2015, 20, 1-13.

11. Blaga, A.M.; Crasmareanu, M. Torse-forming $\eta$-Ricci solitons in almost paracontact $\eta$-Einstein geometry. Filomat 2017, 31, 499-504. [CrossRef]

12. Prakasha, D.G.; Hadimani, B.S. $\eta$-Ricci solitons on para-Sasakian manifolds. J. Geom. 2017, 108, 383-392. [CrossRef]

13. Bagewadi, C.S.; Ingalahalli, G. Ricci solitons in Lorentzian $\alpha$-Sasakian manifolds Acta Math. 2012, $28,59-68$.

14. Blaga, A.M.; Perktaş, S.Y. Remarks on almost $\eta$-Ricci solitons in ( $\varepsilon$ )-para Sasakian manifolds. Commun. Fac. Sci. Univ. Ank. Ser. A1 Math. Stat. 2019, 68, 1621-1628. [CrossRef]

15. Brozos-Vazquez, M.; Calvaruso, G.; Garcia-Rio, E.; Gavino-Fernandez, S. Three-dimensional Lorentzian homogeneous Ricci solitons. Israel J. Math. 2012, 188, 385-403. [CrossRef]

16. Calvaruso, G.; Perrone, D. Geometry of H-paracontact metric manifolds. Publ. Math. Debrecen 2015, 86, 325-346. [CrossRef]

17. Manev, M. Ricci-like solitons on almost contact B-metric manifolds. J. Geom. Phys. 2020, 154, 103734. [CrossRef] 
18. Manev, M.; Staikova, M. On almost paracontact Riemannian manifolds of type $(n, n)$. J. Geom. 2001, 72, 108-114. [CrossRef]

19. Manev, M.; Tavkova, V. On almost paracontact almost paracomplex Riemannian manifolds. Facta Univ. Ser. Math. Inform. 2018, 33, 637-657.

20. Manev, M.; Tavkova, V. Lie groups as 3-dimensional almost paracontact almost paracomplex Riemannian manifolds. J. Geom. 2019, 110, 43. [CrossRef]

21. Satō, I. On a structure similar to the almost contact structure. Tensor (N.S.) 1976, 30, $219-224$.

22. Ivanov, S.; Manev, H.; Manev, M. Para-Sasaki-like Riemannian manifolds and new Einstein metrics. Rev. R. Acad. Cienc. Exactas Fis. Nat. Ser. A Math. RACSAM 2021, 115, 112. [CrossRef]

23. Chow, B.; Lu, P.; Ni, L. Hamilton's Ricci Flow; Graduate Studies in Mathematics 77; AMS Science Press: Providence, RI, USA, 2006. 